\title{
Evaluation of Cyanoacrylate Glue in Mesh Fixation in Non-Incisional Ventral Hernia
}

\author{
MAHMOUD K. ATLAM, M.Sc.; HOSSAM R. MOUSSA, M.D.; MOHAMED A. MLEES, M.D. and \\ AYMAN A. ELNEMR, M.D.
}

The Department of General Surgery, Faculty of Medicine, Tanta University, Egypt

\begin{abstract}
Background: The occurrence of post-operative chronic pain after mesh hernioplasty in nonincisional ventral hernia is especially disturbing as it is felt by $0-43 \%$ of patients, $11 \%$ on average. Post-operative pain is the consequence of tissue dissection and post-operative local inflammation. As a result, the surrounding nerves become damaged and painful stimulation occurs. The cyanoacrylate glue, the characteristic of histoacryl such as efficacy, excellent local tolerability, and less of adverse effects makes it an optimal choice for the atraumatic mesh fixation. It has been used in a variety of surgical fields for its effectiveness, excellent local tolerability and relative lack of adverse effects and contraindications. However, the hospital costs were increased by the use of cyanoacrylate glue instead of the sutures.
\end{abstract}

Aim of Study: The aim of this study is to evaluate the feasibility, efficacy of cyanoacrylate glue in mesh fixation in non-incisional ventral hernia repair as regard the operative time, the post-operative pain, the occurrence of wound seroma and recurrence rate.

Patients and Methods: This prospective study was carried out on 20 patients of different ages in either sex presented with nonincisional ventral hernia who were subjected to mesh hernioplasty with cyanoacrylate glue. They were followedup for chronic pain, seroma, recurrence.

Results: The post-operative pain was evaluated in 1 st month and at 6 months by visual analogue score, 8 out of 20 patients $(40 \%)$ had mild pain and 2 out of 20 patients $(10 \%)$ had moderate pain. Shrinkage of the mesh reported in 2 out of 20 patients $(10 \%)$ and 5 out of 20 patients $(25 \%)$ developed seroma over the mesh that resolved on conservative treatment. No recurrence of the hernia during the follow-up period by clinical examination and by abdominal ultrasound.

Conclusion: The mesh fixation with cyanoacrylate glue in nonincisional ventral hernia is more expensive than using sutures in mesh fixation but, it can reduce the need for analgesics and improve life style by decreasing incidence of chronic post-operative pain. We recommend performing further studies with larger population.

Correspondence to: Dr. Mahmoud K. Atlam, The Department of General Surgery, Faculty of Medicine, Tanta University, Egypt
Key Words: Cyanoacrylate glue - Mesh fixation - Non incisional - Ventrol hernia.

\section{Introduction}

A VENTRAL hernia occurs when a weak spot in the abdomen enables abdominal tissue or an organ (such as an intestine) to protrude through the abdominal wall. These hernias are visibly identified by a bulge in the abdomen. The most common sites for ventral hernias are the site of a previous abdominal procedure (an incisional hernia), umbilical hernia, paraumbilical hernia and epigastric hernia [1]

Tension-free hernioplasty using a variety of techniques and synthetic mesh has become a widely accepted method of management, and has been found to reduce the incidence of recurrence. However, surgeons and patients are currently concentrating on other post-operative measures of the quality of hernia repair [2] .

The occurrence of post-operative chronic pain is especially disturbing as it is felt by $0-43 \%$ of patients, $11 \%$ on average. Post-operative pain is the consequence of tissue dissection and postoperative local inflammation. As a result, the surrounding nerves become damaged and painful stimulation occurs. Surgeon's experience, local complications, synthetic material implantation are considered to be dominant causative factors. Inflammation appears with the ligation of the hernia sack, neurectomy, when a nerve gets captured into a suture or scar around the implant [3].

However, in an attempt to reduce chronic pain induced by sutures, several methods of mesh fixation have been developed over time, including absorbable sutures, self-adhering meshes and glue fixation. The latter two are presumed to prevent 
nerve entrapment and direct trauma to the nerves [4].

The cyanoacrylate glue, the characteristic of histoacryl such as efficacy, excellent local tolerability, and less of adverse effects makes it an optimal choice for the atraumatic mesh fixation. It has been used in a variety of surgical fields for its effectiveness, excellent local tolerability and relative lack of adverse effects and contraindications. However, the hospital costs were increased by the use of cyanoacrylate glue instead of the sutures [5].

The use of cyanoacrylate glue appeared to be promising for mesh fixation in hernia repair, with recurrence rates comparable with those of sutured mesh fixation, and without adhesive-related complications. However, concerns about toxicity and presumed inflammatory reactions to this chemical adhesive have hampered its widespread use. Because of these concerns, N-Butyl-2-Cyanoacrylate (NBCA) was developed, and has been reported to have reduced toxicity and fewer inflammatory reactions [6].

\section{Patients and Methods}

This prospective study was carried out on 20 patients of different ages in either sex presented with ventral hernia at the Surgical Oncology Unit, General Surgery Department, Tanta University Hospital, during the period from August 2017 to July 2018.

\section{Inclusion criteria:}

Patients aged $\geq 18$ year, patients presented with nonincisional epigastric hernia, paraumbilical hernia, or umbilical hernia, patients presented with hernial defect less than 5 cmwere included in this study.

\section{Exclusion criteria:}

1- Patients have divarication of recti.

2- Patients with complicated hernia: Irreducible, obstructed or strangulated hernia.

3- Patients with incisional hernia.

Informed consent was obtained from all patients after detailed description of the procedure before entry to the study. The study was approved by Ethical Committee of Tanta University, Faculty of Medicine.

\section{Every patient was subjected to:}

1- Full history with considerable emphasis on:

- Age, sex, residence and occupation.
- Special habits of medical importance e.g. smoking.

- Medical conditions e.g. diabetes mellitus, hypertension, liver diseases, lung diseases and urinary diseases.

- Past history of previous surgery.

A particular stress upon risk factors of ventral hernia as smoking, the nature of patients work specially heavy weight lifting jobs, chronic cough, benign prostatic hyperplasia, chronic constipation, Body Mass Index (BMI) >25 and multiple pregnancies.

2- Clinical examination: General: Vital data (temperature, pulse, blood pressure and respiratory rate) and general condition.

\section{3- Investigations:}

A- Complete blood count, liver function tests, renal function tests, fasting and postprandial blood glucose levels.

B- Chest X-ray: To detect chest infections, chronic obstructive pulmonary diseases, etc.

C- Pelvi-abdominal ultrasonography: To detect defect size, contents of the hernial sac and predisposing factor as: Organomegaly, intra-abdominal masses, benign prostatic hyperplasia or ascites.

Pre-operative preparation: Prophylactic antibiotics using a combination of amoxicillin and clavulanic acid were routinely administrated by intravenous route 2 hours before induction of anesthesia.

\section{Surgical procedure:}

The procedure was performed under general endotracheal anesthesia in supine position. Skin disinfection was done by $10 \%$ povidone iodine antiseptic solution.

A transverse elliptical incision over the hernia was made. The skin edges of both sides were hold using Allis clamps. The incision was deepened through sub-cutaneous fat and aponeurosis and dissection was continued till identification of the margins of the hernial defect circumferentially, down to the hernial neck. Then, the sac was dissected from fatty tissue and area of rectus sheath (the white tough tissue) around it. The defect was closed anatomically with a continuous polyprolene suture (prolene-1).

Tailoring and spreading properly the polyprolene mesh ${ }^{\circledR}$ (Ethicon) was done. The mesh was fixed by cyanoacrylate glue (Histoacryl®-B. Braun) 
Fig. (1) onto the underlying tissue as follow: The ampoule was opened by twisting its tip, then cyanoacrylate glue was applied on the edges of mesh first followed by the centre of mesh in a drop-wise manner Fig. (2).

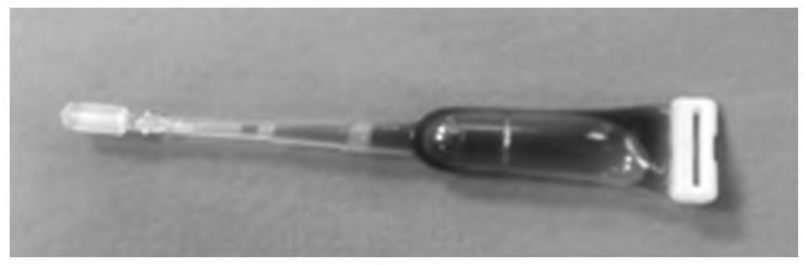

Fig. (1): Ampoule of Histoacryl®-B. Braun.

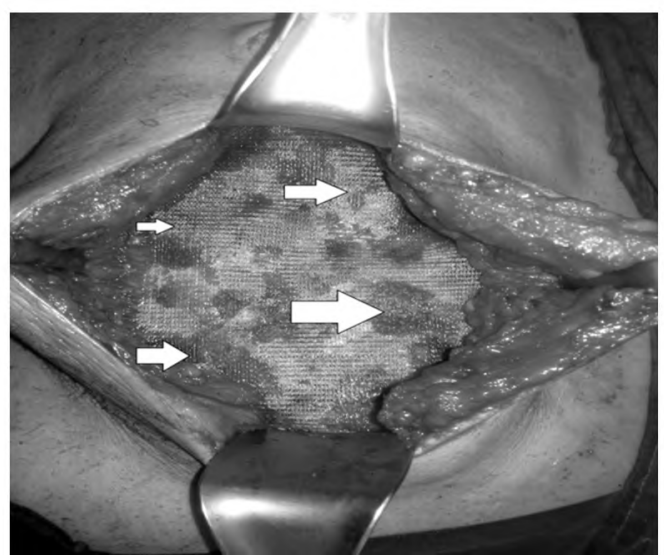

Fig. (2): The mesh fixed by drops of Histoacryl.

During the procedure, the tip of ampoule may gradually clog up causing spitting or complete obstruction. At this moment cutting of the tip at lower level was done and light pressure was applied to the ampoule. This problem was avoided by keeping some distance between the tip of ampoule and the mesh. Usually, one ampoule of histoacryl was used to fix the mesh.

A tube of suction drain was put above the mesh. The subcutaneous layer was closed with interrupted absorbable sutures (vicryl-2/0) and skin was closed with subcuticular sutures using prolene 2-0.

Post-operatively, all patients were given a combination of oral amoxicillin and clavulanic acid every 12 hours and oral diclofenac sodium 50mg on need. They were discharged on the second postoperative day.

\section{Follow-up:}

Clinical evaluation during the follow-up period was done either by telephone call or in an outpatient setting. The patients were followed-up weekly in the first month and then after 3 month and after 6 months. Patients were instructed to contact the surgical staff if any symptoms appeared between the scheduled visits or at any time after the dis- charge day. The drain was removed when the amount of drain fluid reached less than $50 \mathrm{cc} / 24$ hours or maximum of 10 days.

Early post-operative complications were recorded within 3 months including seroma, hematoma, Surgical Site Infection (SSI), pain, and time to return to work (for patients who did not work, we evaluated the return to normal activity like walking, driving and sports). Seroma formation is defined as the accumulation of sterile serous fluid within the spaces created during herniorraphy [7]. Hematoma was defined as a localized collection of blood in the dissected surgical planes [8]. Surgical site infection can be superficial or deep. Superficial SSI is defined as this infection occurs just in the area of the skin where the incision was made. Deep SSI is defined as infection that occurs beneath the incision area in muscle and the tissues surrounding the muscles [9].

Early post-operative pain was evaluated during the first 3 months. The pain was evaluated by Visual Analogue Scale (VAS). It is unidimensional measure of pain intensity, which has been widely used in diverse adult populations. It is a continuous scale comprised of a horizontal (HVAS), usually 10 centimeters $(100 \mathrm{~mm})$ in length, anchored by 2 verbal descriptors, one for each symptom extreme.

For pain intensity, the scale is most commonly anchored by "no pain" (score of 0 ) and "pain as bad as it could be" or "worst imaginable pain".

The pain VAS was self-completed by the patient who asked to place a line perpendicular to the VAS line at the point that represents their pain intensity. Using a ruler, the score is determined by measuring the distance $(\mathrm{mm})$ on the $10-\mathrm{cm}$ line between the "no pain" anchor and the patient's mark, providing a range of scores from 0-100. A higher score indicates greater pain intensity. The pain intensity was marked as: None, mild, moderate, or severe, the following cut points on the pain VAS have been recommended: No pain $(0-4 \mathrm{~mm})$, mild pain $(5-44$ $\mathrm{mm})$, moderate pain $(45-74 \mathrm{~mm})$, and severe pain (75-100mm). An analgesic as oral diclofenac sodium was given for mild pain, intravenous analgesics as ketorolac was given for moderate pain; opioid analgesic was reserved for severe pain.

Long term complications were focused on chronic pain, shrinkage or migration of mesh, and recurrence.

Chronic pain was defined by the International Association for the Study of Pain (IASP) as pain that persists after the normal healing process, 
typically 3 months after surgery [10]. However, this definition has been modified lately into pain that is a direct consequence of nerve lesion or a disease affecting the somatosensory system in patients who did not have pain prior to an original hernia surgery or, if they did, whose pain is different compared with that experienced pre-operatively, present more than 3 months [11].

Mesh shrinkage means reduction of size of mesh due to retraction of the fibrous scar tissues around the mesh [12].

Follow-up by ultrasonography was done serially at 2 weeks, 3 months, and 6 months post-operatively to detect mesh shrinkage, or migration, and recurrence of hernia.

\section{Statistical analysis of the data:}

Data were collected, tabulated and analyzed using IBM SPSS computer software package Version 20.0. Qualitative data were described using number and percent. The kolmogorov-smirnov test was used to verify the normality of distribution. Quantitative data were described using range (minimum and maximum), mean, standard deviation and median.

\section{Results}

This prospective study was carried out on 20 patients with nonincisional ventral hernia at the Surgical Oncology Unit, General Surgery Department, Tanta University Hospital, during the period from August 2017 to July 2018.

Demographic characteristics of studied patients:

Table (1): Distribution of the studied cases according to demographic data $(n=20)$.

\begin{tabular}{ccc}
\hline & No. & $\%$ \\
\hline Sex: & & \\
Male & 7 & 35.0 \\
Female & 13 & 65.0 \\
Age (years): & & \\
$<40$ & 17 & 85.0 \\
$\geq 40$ & 3 & 15.0 \\
\hline
\end{tabular}

Predisposing factors of hernia and comorbiditieis:

In (Table 2), the risk factors and co-morbidities of studied patients are shown. The Body Mass Index (BMI) of obese patients ranged from 26 to 31 with a mean of $28 \pm 1.3$. There were four male smokers and the smoking index of two of them were 300 and 400 and they were complaining of chronic cough and received medical treatment and advised to stop smoking 2 weeks before time of operation while the smoking index of the other two patients were 80 and 100 . All the 13 females were multigravida and the number of pregnancies ranged from 2 to 4 times with mean $2.6 \pm 0.65$ times. Six out of 20 patients underwent caesarean section; three patients underwent caesarean section and appendectomy.

Table (2): Distribution of the studied cases according to risk factors $(n=20)$

\begin{tabular}{lll}
\hline & No. & $\%$ \\
\hline Obesity & 12 & 60 \\
Smoking: & & \\
No & 16 & 80.0 \\
Yes & 4 & 20.0 \\
Co-morbidities: & & \\
No & 16 & 80.0 \\
Hypertensive & 2 & 10.0 \\
Diabetic & 2 & 10.0 \\
Surgical history: & & \\
No & 11 & 55.0 \\
Yes & 9 & 45.0 \\
\hline
\end{tabular}

Type of hernia and ultrasound findings:

Table (3): Distribution of the studied cases according to size of defect $(\mathrm{cm})$ as detected by ultrasonography $(\mathrm{n}$ $=20$ ).

\begin{tabular}{lcc}
\hline Size of defect $(\mathrm{cm})$ & No. & $\%$ \\
\hline$<2 \mathrm{~cm}$ & 10 & 50.0 \\
$\geq 2 \mathrm{~cm}$ & 10 & 50.0 \\
Min.-max. & $0.70-3.0$ \\
Mean \pm SD. & $1.75 \pm 0.75$ \\
Median & \multicolumn{2}{c}{1.85} \\
\hline
\end{tabular}

Table (4): Distribution of the studied cases according to content of hernial sac as detected by ultrasonography $(n=20)$.

\begin{tabular}{lcc}
\hline Content of hernial sac in ultrasound & No. & $\%$ \\
\hline Omentum only & 10 & 50.0 \\
Bowel loops and omentum & 8 & 40.0 \\
Bowel loops only & 2 & 10.0 \\
\hline
\end{tabular}

The time of mesh fixation and time of operative time:

Table (5): Distribution of the studied cases according to time of operation and time of mesh fixation $(n=20)$.

\begin{tabular}{lll}
\hline & No. & $\%$ \\
\hline Time of mesh fixation: & & \\
$\quad$ 5 minutes & 14 & 70.0 \\
$\quad>5$ minutes & 6 & 30.0 \\
Time of operation: & & \\
$<60$ & 14 & 70.0 \\
$\geq 60$ & 6 & 30.0 \\
\hline
\end{tabular}




\section{Post-operative complications:}

In the present study, there was no intraoperative complication and no mortality. One of the most important parameters in evaluating cyanoacrylate glue was the post-operative pain. In the first month, 8 out of 20 patients $(40 \%)$ had mild pain (VAS= $0 \sim 4)$ and $75 \%$ of these patients were females, $25 \%$ of these patients had seroma over the mesh. The pain was relieved by oral diclofenac sodium 50 mg every 12 hours for 5 to 7 days. Two female patients $(10 \%)$ had moderate pain (VAS=5 44) and one of them had shrinkage of mesh in the ultrasonography in the follow-up. Their pain were controlled by intravenous ketorolac 50mg every 12 hours for 3 days then replaced by oral diclofenac sodium 50mg for 7 days. After 3 and 6 months, no patients had complained of chronic pain (Table 6).

Table (6): Distribution of the studied cases according to postoperative pain $(n=20)$.

\begin{tabular}{llclc}
\hline \multirow{2}{*}{ Post-operative pain } & \multicolumn{2}{c}{$1-3$ months } & \multicolumn{2}{c}{$3-6$ months } \\
\cline { 2 - 5 } & No. & $\%$ & No. & $\%$ \\
\hline No (VAS=0) & 10 & 50.0 & 20 & 100.0 \\
Mild (VAS=0-4) & 8 & 40.0 & 0 & 0.0 \\
Moderate (VAS=5-44) & 2 & 10.0 & 0 & 0.0 \\
\hline
\end{tabular}

In the present study, as regard the hospital stay, 18 out of the 20 patients $(90 \%)$ stay in the hospital for one day and the remaining two patients (10\%) stay for 2 days as they were complaining of postoperative cough for which they received medical treatment. The time of removal of the drain ranged from $3 \sim 10$ days with a mean of $7 \pm 2.4$ days. The mean time to return to work was $20 \pm 2.4$ days. Five patients developed seroma that was managed conservatively. All of these patients were obese. One out of the 20 patients (5\%) developed Superficial SSI which was treated conservatively with antibiotic and daily dressing and improved with the treatment but no patients had deep SSI (Table 7).

Table (7): Distribution of the studied cases according to postoperative complications.

\begin{tabular}{|c|c|c|c|}
\hline \multicolumn{2}{|l|}{ Complications } & \multirow{2}{*}{$\frac{\text { No. }}{5}$} & \multirow{2}{*}{$\frac{\%}{25.0}$} \\
\hline Seroma: & Yes & & \\
\hline & No & 15 & 75.0 \\
\hline \multirow[t]{2}{*}{ Superficial SSI: } & Yes & 1 & 5.0 \\
\hline & No & 19 & 95.0 \\
\hline \multirow[t]{2}{*}{ Shrinkage: } & Yes & 1 & 5.0 \\
\hline & No & 19 & 95.0 \\
\hline \multirow[t]{2}{*}{ Migration: } & Yes & 0 & 0.0 \\
\hline & No & 20 & 100.0 \\
\hline \multirow[t]{2}{*}{ Recurrence: } & Yes & 0 & 00.0 \\
\hline & No & 20 & 100.0 \\
\hline
\end{tabular}

\section{Discussion}

The nonincisional ventral hernia is the second most common of abdominal hernia after inguinal hernia; it represents about $10 \%$ of abdominal hernia. It can be umbilical, paraumbilical, epigastric or spegelian hernia [5]

The definitive treatment of ventral abdominal wall hernia is the surgical repair to avoid incarceration, obstruction or strangulation if left without repair. Trusses or belts -made to apply pressure on hernia-require correct fitting. When used correctly, part or complete control of hernia was achieved in $31 \%$ of patients, $64 \%$ of patients found it uncomfortable, and complications occur in $4 \%$ of patients [6]

Cyanoacrylate glue is synthetic cyanoacrylatebased compounds similar to 'Superglue', which work by contact-induced exothermic hydroxylation of the monomer to form a stable polymer80. During the Vietnam War (1955-1975) many soldiers carried 'Superglue' for quick equipment repairs on the battlefield. Inevitably, when soldiers were wounded, bleeding and necessity called. It was soon discovered that cyanoacrylate glue had an exceptional ability to hold wounds closed, as it polymerized and hardened when exposed to moisture. Eventually field surgeons started spraying it on wounds before transit to army hospitals and many lives were saved as a result. Despite promising results, the study received criticism related to the local production of heat when using the glue and possible nerve and tissue damage as a result [7] .

More recently, De Goede B [9] performed a systematic review of 38 publications assessing the use of Cyanoacrylate glue in hernia repair. They concluded that Cyanoacrylate glue was an efficacious alternative for mesh fixation compared to mechanical fixation devices. The use of Cyanoacrylate glue has also been advocated by Eklund A [10] in a systematic review which included 36 studies assessing cyanoacrylate glue in a total of 5,993 patients undergoing various abdominal wall hernia repairs. Cyanoacrylate glue was compared favorably with traditional methods of mesh fixation, being associated with shorter operative times and hospital stays and a lower incidence of chronic pain.

In the present study, the patients' age ranged from 30 to 45 years with a mean of 34.50 \pm 4.07 years. This result agrees with Lee MG et al., 84 who recorded ages of patients ranged from 25 to 48 with a mean of $37.50 \pm 3.04$ years, in contrast to our study, Brugger et al., [12] found the mean 
age of $57.50 \pm 10.5$ years. Similarly, Ming-Gang W et al., [13] reported that the mean age of patients were $61.2 \pm 11.8$ years.

In our study, 13 out of the 20 patients $(65 \%)$ were females while 7 patients $(35 \%)$ of patients were males. This agrees with Lee MG et al., [11] who reported that $70 \%$ of patients were females and 30\% were males. While De Goede B [9] reported that $60 \%$ of patients were males and $40 \%$ of patients were females. This discrepancy can be explained that most of our patients were females who were obese and had multiple pregnancies.

In the present study, there was a strong relation between the number of pregnancies, obesity and the incidence of ventral hernia. All female patients had previous pregnancies with minimum 2 times and maximum 4 pregnancies with mean $2.6 \pm 0.65$ times and median 3 times. Twelve out of the 20 patients $(60 \%)$ were obese. This agrees with of the results of Lee MG et al., [11] who reported that $80 \%$ of female patients had previous pregnancies and the number of pregnancies ranged from minimum one time and maximum 4 pregnancies with a mean of $2.0 \pm 0.65$ times and median 2 times. They also reported that $50 \%$ of patients were obese.

In the current study, 15 out of the 20 patients $(75 \%)$ had paraumbilical hernias and 5 patients $(25 \%)$ had epigastric hernias. This agrees with Kalpesh Jani87 who reported that $70 \%$ of patients had paraumbilical hernia, $30 \%$ of patients had epigastric hernia and $10 \%$ of patients had umbilical hernia. Also, Chevrel JP et al., [15] reported that $60 \%$ of patients had paraumbilical hernia, $20 \%$ of the patients had epigastric hernia and $10 \%$ had umbilical hernia. On the other hand, C. Kim-Fuchs et al., [16] reported that $50 \%$ of their patients had umbilical hernia, $40 \%$ had paraumbilical hernia and $10 \%$ had epigasrtic hernia.

In our study, the content of hernial sac was omentum in 10 out of the 20 patients $(50 \%), 8$ patients $(40 \%)$ there were omentum and bowel loops and in 2 patients $(10 \%)$ there were only bowel loops. This comes in agreement with Chevrel JP et al., [15] who reported that $60 \%$ of their patients had omentum in the hernia sac, $35 \%$ had omentum and bowel loops and only $5 \%$ had just bowel loops. However, Kalpesh Jani [14] reported that $60 \%$ of their patients had bowel loops and omentum in the hernial sac, $40 \%$ had only omentum.

In our patients, the size of hernial defect ranged from 0.7 to $3 \mathrm{~cm}$ with a mean of $1.75 \pm 0.75 \mathrm{~cm}$. As the size of defect was limited to less than $5 \mathrm{~cm}$ in this study, we were able to fix the mesh with only one ampoule of Histoacryl $\AA-B$. Braun which is expensive. This result agrees with the results of Chevrel JP et al., [15] who reported that one of the drawbacks of tissue adhesives that they are economically expensive.

In this work, mesh fixation had done in a short time that was 2-10 minutes with a mean of $3.95 \pm$ 2.04 minutes and median 3.0 minutes, and the operation time was 45-90 minutes with a mean of $59.50 \pm 13.85$ minutes and median 57.50 minutes. This is parallel to the study of Eklund A [10] who recorded that the time of mesh fixation was 3-9 minutes with a mean of $3.55 \pm 1.64$ minutes and median 4 minutes and the operation time was 40100 minutes with a mean of $56.50 \pm 19.85$ minutes. Also, this is in agreement with the results of De Goede B [9] who reported that the time of mesh fixation was 4-9 minutes with a mean of $4.45 \pm 2.34$ minutes and median 6 minutes and the operation time was 50-100 minutes with a mean of $61.23 \pm$ 10.85 minutes.

In our study, 18 out of the 20 patients (90\%) stay in the hospital one day and 2 out of the 20 patients (10\%) stay 2 days. This agrees with results of Olmi et al., 90 who reported that all patients were discharged the day after surgery, also in Topart et al., 91 study, the mean post-operative hospitalization was one day. However, in Ming-Gang W et al., 86 study, the length of hospital stay was $3 \pm 0.81$ days.

In our study, the time of removal of the drain was 3-10 days with a mean of $7 \pm 2.4$ days. This agrees with the results of C. Kim-Fuchs et al., 89 who reported that the time of removal the drain ranged from 2 days to 8 days with a mean of $7 \pm 0.4$.

In the current study, the mean time to return to work was $20 \pm 2.4$ days. Similarly, in Arthur et al., 92 study, the mean time to return to work was $21 \pm 4.3$ days. Also, this agrees with results of Ming-Gang W et al., 86 who reported that the mean time to return to work was $21.5 \pm 2.2$.

In the present study, one out of the 20 patients (5\%) developed only superficial wound infection which improved by antibiotic and daily dressing. Similarly, in Arthur et al., 92 study, 5.3\% of the patients presented with mild fever and localized pain in the wound. Ciprofloxacin was given for suspected infection that resolved without further intervention. However, in Ming-Gang W et al., 86 study, there was no wound infection. Also, in Topart et al., 91 study, no fever or inflammation was reported after surgery. 
In our study, early post-operative pain was evaluated weekly during the 1 st month then at 2 nd and 3 rd month and by visual analogue score. Chronic pain was defined as pain persisting after 3 months and it was evaluated at $3 \mathrm{rd}$ and 6 th month. Eight out of the 20 patients (40\%) had mild pain during first month that was relieved by oral diclofenac sodium; 50mg every 12 hours for 5-7 days. Two out of the 20 patients $(10 \%)$ had moderate pain that was relieved by intravenous Ketorolac; 50mg every 12 hours for 3 days and continue on oral analgesics. After 6 months, no patients were complaining of chronic post-operative pain. This agrees with the results of Kalpesh Jani [14] who reported that $30 \%$ of the patients had early pain in the first month, and no patients had chronic pain after 6 th month. Also, this agrees with the results of De Goede B [9] who reported that after 1 month $10 \%$ had pain, and 5\% after month. Also, this agrees with the results of Giovanni T et al., 93 who reported that $50 \%$ of patients had early post-operative pain in first month and $7.7 \%$ had chronic pain after 6th month.

In this report, Shrinkage of the mesh was reported in 1 out of the 20 patients (5\%). This comes in agreement with results of Paola Losi et al., [21] who reported mesh shrinkage in 3\% of patients. Also, this comes in agreement with results of C. Kim-Fuchs et al., 90 who reported mesh shrinkage in $4 \%$ of patients.

In the current study, five out of the 20 patients (25\%) developed seroma over the mesh that resolved by conservative measures. This is in agreement with the results of Paola Losi et al., [21] who reported that seroma developed in $20 \%$ of their patients that was managed by prophylactic antibiotics and anti-inflammatory drugs, and no patients need intervention.

In our study, there was no recurrence of the hernia during the follow-up period by clinical examination and abdominal ultrasonography. This agrees with the results of C. Kim-Fuchs et al., [16] who reported no recurrence of the hernia developed during the following-up period for 6 months. Also, it agrees with the results of Kalpesh Jani [14] who did not record recurrence of the hernia in the following-up period for 12 months. However, this follow-up period is not enough and longer followup to confirm and support our results.

\section{Conclusion:}

The mesh fixation with cyanoacrylate glue in nonincisional ventral hernia is easy, feasible with no chronic post-operative pain, minimal complica- tions and no recurrence of the hernia. It is more expensive than using sutures in mesh fixation but, it can reduce the need for analgesics and improve life style by decreasing incidence of chronic postoperative pain. We recommend performing further studies with larger population scale and longer follow-up with control group to confirm and support our results.

\section{References}

1- A. ARROYO, P. GARCÍA, F. PÉREZ, et al.: Randomized clinical trial comparing suture and mesh repair of umbilical hernia in adults Br. J. Surg., 88: 1321-3, 2001.

2- V. SHANKARAN, D.J. WEBER, L. REED, et al.: Review of available prosthetics for ventral hernia repair. Ann. Surg., 253: 16-26, 2011.

3- M. CEVASCO and K.M.F. ITANI: Ventral hernia repair with synthetic, composite, and biologic mesh: Characteristics, indications, and infection profiles Surg. Infect. (Larchmt), 13: 209-15, 2012.

4- NEGRO P., BASILE F., BRESCIA A., et al.: Open tensionfree Lichtenstein repair of inguinal hernia: Use of fibrin glue versus sutures for mesh fixation. Hernia, (7): 15-4, 2011.

5- WILLIAMS N.S., BULSTRODE C.J.K. and O'CONNELL P.R.: Bailey \& Love's Short Practice of Surgery. 25 th edn London: Hodder Arnold, 26: 334-6, 2008.

6- CHEEK C.M., WILLIAMS M.H. and FARNDON J.R.: Trusses in management of hernia today. British Journal of Surgury, 82: 1611-3, 1995.

7- HAWKER, GILLIAN A., MIAN S., et al.: Measures of adult pain: Visual analog scale for pain (vas pain), numeric rating scale for pain (nrs pain), mcgill pain questionnaire (mpq), short-form mcgill pain questionnaire (sf-mpq), chronic pain grade scale (cpgs), short form-36 bodily pain scale (sf-36 bps), and measure of intermittent and constant osteoarthritis pain (icoap)." Arthritis Care \& Research, 63: 11, 2011.

8- SHEN Y.M., SUN W.B., CHEN J., et al.: NBCA medical adhesive (n-butyl-2-cyanoacrylate) versus suture for patch fixation in Lichtenstein inguinal herniorrhaphy: A randomized controlled trial. Surgery, 151: 550-5, 2012.

9- De GOEDE B., KLITISIE P.J., VAN KEMPEN B.J., et al.: Meta-analysis of glue versus sutured mesh fixation for Lichtenstein inguinal hernia repair. Br. J. Surg., 100 (6): 735-42, 2013.

10- EKLUND A., MONTGOMERY A., BERGKVIST L. and RUDBERG C.: Swedish multicentre trial of inguinal hernia repair by laparoscopy (SMIL) study group. Chronic pain 5 years after randomizedcomparison of laparoscopic and Lichtenstein inguinal hernia repair. Br. J. Surg., 97 (4): 600-8, 2010.

11-LEE M.G., WILDISEN A., HAMEL C., et al.: Randomized clinical trial of Lichtenstein's operation versus mesh plug for inguinal hernia repair. Br. J. Surg., 94: 36-41, 2007.

12- BRUGGER, JONES D., ROBERTS A.C., et al.: Applications of fibrin sealant in surgery. Surg. Innov., 12 (3): 203-13, 2005 
13- MING-GANG WANG, DREW P.J., QURESHI A., et al.: Preliminary experience with butyl-2-cyanoacrylate adhesive in tension-free inguinal hernia repair. Br. J. Surg., 83 (8): 1100, 1996.

14- KALPESH JANI: The use of fibrin glues in the surgical treatment of incisional hernias. Hernia, 1 (1): 9-14, 2010.

15- CHEVREL J.P., BARRANCO A., SOCAS M., et al.: Systematic review of the use of fibrin sealant in abdominalwall repair surgery. Hernia, 15 (4): 361-9, 2011.

16- C. KIM-FUCHS, E. ANGST, S. VORBURGER, et al.: Prospective randomized trial comparing sutured with sutureless mesh fixation for Lichtenstein hernia repair: Long-term results. Hernia, 16: 21-7, 2012.

17- TOPART P., VANDENBROUCKE F. and LOZAC'H P.: Tisseel versus tack staples as mesh fixation in totally extraperitoneal laparoscopic repair of groin hernias: A retrospective analysis. Surg. Endosc., May, 19 (5): 7247, 2005.
18- OLMI S., CESANA G., SAGUTTI L., et al.: Laparoscopic incisional hernia repair with fibrin glue in select patients. JSLS, 14 (2): 240-5, 2010.

19- SASCHA A. MÜLLER, RENE WARSCHKOW, ULRICH BEUTNER, et al.: Use of human fibrin glue (Tisseel) versus staples for mesh fixation in laparoscopic transabdominal preperitoneal hernioplasty (TISTA): A randomized controlled trial. BMC Surgery, 14: 18, 2014.

20- GIOVANNI D. TEBALA, VALERIA TOGNONI, ZOE TRISTRAM, et al.: Cyanoacrylate Glue versus Suture Fixation of Mesh in Inguinal Hernia Open Repair: A Randomized Controlled Clinical Trial. Gastroenterol Hepatol Open Access, 2 (5): 54-8, 2015.

21- PAOLA LOSI, BURCHIELLI S., SPILLER D., et al.: Cyanoacrylate surgical glue as an alternative to suture threads for mesh fixation in hernia repair. J. Surg. Res., 163 (2): 53-8, 2010.

\title{
تقييم دور صمح سيانوآكريلات فى تثبيت الشبكة

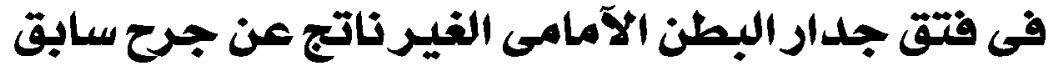

\author{
المقدمة: يحدث فتق جدار البطن الآمامى مثل الفتق السرى والفتق حل السرة والفتق الثرسوفى نتيجة لآسباب كثيرة منها: الحمل المتكرد

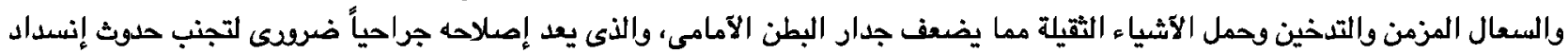

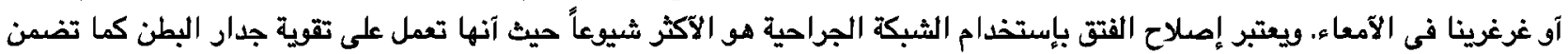

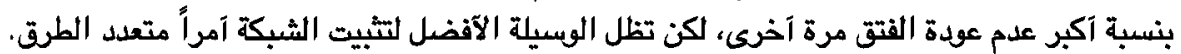

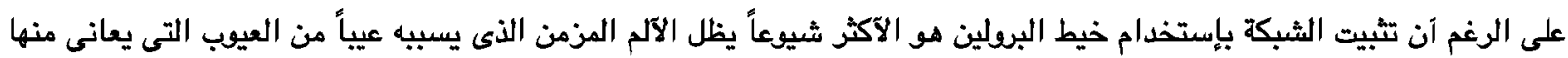

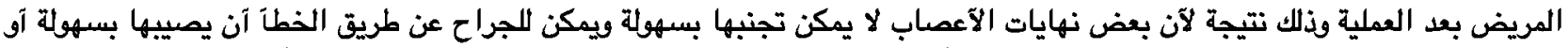

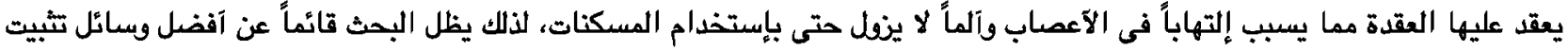

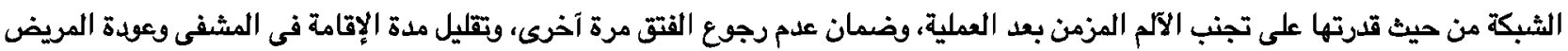 \\ اللعمل بآسرع وقت ممكن.

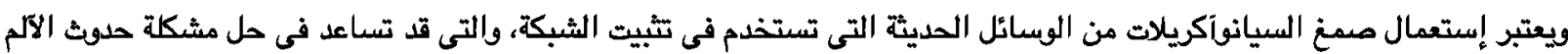

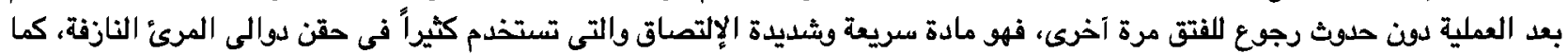 \\ يستخدمه البعض آحياناً في غلق جرع الجق هرة الجلد لهن الحاجة إلى الخياطة. \\ وعلى الرغم من آن هنالك مخاوف كثيرة حول قدرة صمن السيانوآكريلات على تثييت الشبكة، وإحتمالية حلوث رجوع الفتق فى حالة إستخدامه،

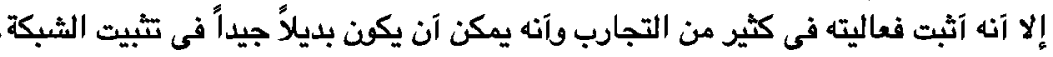

